\title{
A SYSTEMATIC REVIEW OF OBSERVATIONAL STUDIES ASSESSING THE IMPACT OF OXIDATIVE STRESS IN COGNITIVE DECLINE
}

DOI: 10.36740/WLek202108137

\author{
Ioanna V. Papathanasiou' ${ }^{1}$ Evangelos C. Fradelos ${ }^{1}$, Foteini Malli' ${ }^{1}$ loannis Stefanidis ${ }^{2}$, Elias Zintzaras ${ }^{2,3}$, \\ Chrysoula Doxani \\ 'FACULTY OF NURSING, UNIVERSITY OF THESSALLY, THESSALLY, GREECE \\ 2FACULTY OF MEDICINE, UNIVERSITY OF THESSALY, THESSALY, GREECE \\ ${ }^{3}$ CENTER FOR CLINICAL EVIDENCE SYNTHESIS, THE INSTITUTE FOR CLINICAL RESEARCH AND HEALTH POLICY STUDIES, TUFTS MEDICAL CENTER, TUFTS \\ UNIVERSITY SCHOOL OF MEDICINE, BOSTON, MA, UNITED STATES
}

\begin{abstract}
Introduction: Cognitive functions are defined as the mental processes through which information is received, processed, stored, and retrieved. Oxidation is considered as an important factor that affects negatively the brain function.

The aim: To assess the impact of oxidative stress, as it is measured by oxidative markers or by the presence of anti-oxidants, on cognitive decline.

Materials and methods: A systematic review of published observational studies in PubMed and in Scopus was performed. During the review process the keywords were used as follows: ("oxidative stress") AND ("cognitive decline" OR "cognitive dysfunction" OR "cognitive impairment" OR "cognitive deficiency") AND ("observational study" OR "cross sectional study" OR "prospective study" OR "retrospective study" OR"cohort study"). The search was conducted for the years from 2016 to 2020.

Conclusions: Seventy-four eligible studies were identified. Thirteen studies met the inclusion and quality criteria and were included in the systematic review. The studies conducted in ten different countries. Information about oxidative stress biomarkers is available in eight studies, while information about antioxidant factors is in ten studies. In all the thirteen studies the cognitive function was assessed with specific tools - scales. In the majority of studies, the presence of high scores in oxidative markers was positively associated with cognitive decline, while higher levels of antioxidant markers were associated with better cognitive function. Our results indicate that oxidative stress may be significantly associated with cognitive decline. The presence of the antioxidants glutathione, uric acid, melatonin, cysteine and peroxide dismutase has a positive effect on cognitive function.
\end{abstract}

KEY WORDS: cognitive dysfunction, 0xidative stress, cognitive impairment, observational studies, cognitive function, cognitive decline

Wiad Lek. 2021;74(8):1995-2003

\section{INTRODUCTION}

Cognitive functions are defined as the mental processes through which environmental information is received, selected, processed, stored, and retrieved [1]. The higher cognitive functions through which understanding and interaction with the world take place are usually studied as separate entities, but in reality these functions are in constant connection with each other and sometimes overlap [2]. The main highest cognitive functions are attention, memory, executive functions, speech, visual-spatial and visual-perceptual functions, through which space is analyzed and understood in its three dimensions [3].

As people get older, many experiences a decline in one or more of the cognitive functions, such as memory or the speed of cognitive perception. The degree of cognitive impairment varies between individuals and this is primarily due to differences in the functioning of individual human systems, which are genetically predetermined, but also environmentally affected [4]. Studying the population of the elderly, one can distinguish the following groups, based on normal or non-normal cognitive function: a) seniors who retain all their cognitive functions until the end of their lives, b) seniors who have a minimal cognitive decline c) the elderly who have a mild cognitive impairment (MCI), which is a neurodiagnostic disorder that is divided into two subtypes, amnesic (where deficits are observed in memory) and non-amnesic (where deficits observed in other cognitive functions, such as executive function, attention, visual-spatial and visual-perceptual ability), d) the elderly who are diagnosed with dementia and who over time show a great reduction in mental functions and difficulty in performing basic daily activities [5]

The cognitive functions that usually show a decline in old age are mainly the short-term memory, the ability to encode and retrieve information, the ability to focus or shift attention, the information processing speed, the visual-spatial functions, the orientation, the executive function; and in some diseases, such as Alzheimer's disease, there may be a decrease in language function and communication skills [6]. As life expectancy is high in most countries and more people are moving into old age, cognitive impairment is expected to be a major public and community health problem in the future [7]. Many researchers have pointed out that mild cognitive impairment pre-exists several years before dementia occurs and is usually undiagnosed. Due to the fact that even today there is no effective treatment for dementia, the researchers point out that emphasis should be given on the early diagnosis of mild cognitive impairment 
and on the identification of the factors associated with the pathogenesis and faster progression of mild cognitive impairments [8]. Oxidative stress is considered as an important factor that affects the body substrate causing cognitive impairment [9]. Oxidative stress is defined as the imbalance between the production of reactive oxygen species (ROS) and the ability of a biological system to inactivate these toxic molecules and repair the damage they cause [10]. The homeostasis between oxidative and antioxidant mechanisms is referred to as redox regulation. Reactive oxygen species damage many components of the cell, including proteins, lipids and DNA [11]. Thus, oxidative stress is involved in the occurrence of many diseases over time, such as cancer, cardiovascular disease, atherosclerosis, hypertension, diabetes mellitus, inflammation, inflammation, bipolar disorder, accelerated aging, neurodegenerative diseases and chronic fatigue syndrome [12]. Recent studies have been conducted that aimed to investigate oxidation and age-related cognitive impairment and dementia, in order to determine the predictors of cognitive decline [13,14]. Emphasis was also given on research into the effect of antioxidants on cognitive functions $[14,15]$.

\section{THE AIM}

The present study aims to assess the impact of oxidative stress, as measured by oxidative biomarkers or by the presence of anti-oxidants, on cognitive decline, through the systematic review of observational studies findings, taking under consideration the methodological quality of each specific study.

\section{MATERIALS AND METHODS}

To investigate the impact of oxidative stress on cognitive decline, a systematic review of published observational studies was performed in the PubMed and Scopus da-
Studies identified through PubMed searching (n = 23)

Studies after duplicates removed $(\mathrm{n}=74)$
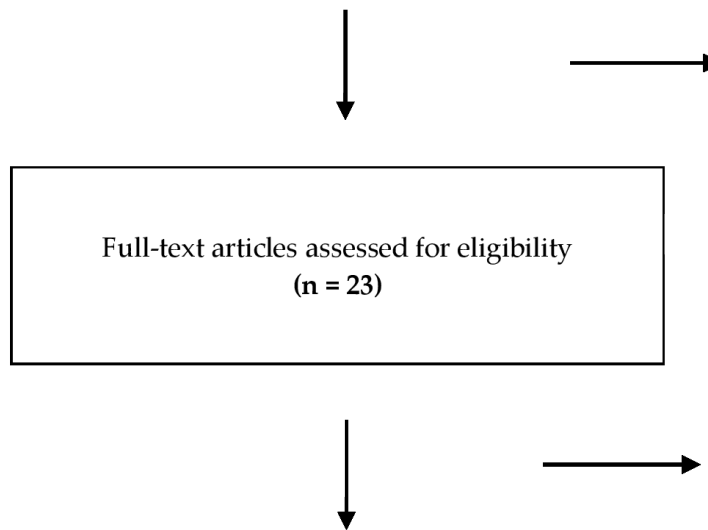

Studies included in the systematic review $(n=13)$

\section{Inclusion criteria}

1. English language-based publication

2. Year of publication 2016 to 2020

3. Population - based sample

4. Observational studies (cohort studies, patient-control studies, cross-sectional studies)

5. Measurement of oxidative stress with one or more oxidative biomarkers or antioxidants

6. Assessment of cognitive function with one or more measurement scales

Studies excluded due to title and abstract not fulfilling inclusion criteria (see box)

$$
\text { (n= 51) }
$$

\section{Studies excluded for not fulfilling:}

a) the inclusion criteria (see box)

b) the AXIS \& CASP quality criteria for observational studies $(\mathrm{n}=10)$

Fig. 1. Flow diagram of the selection process of studies for the systematic review. 


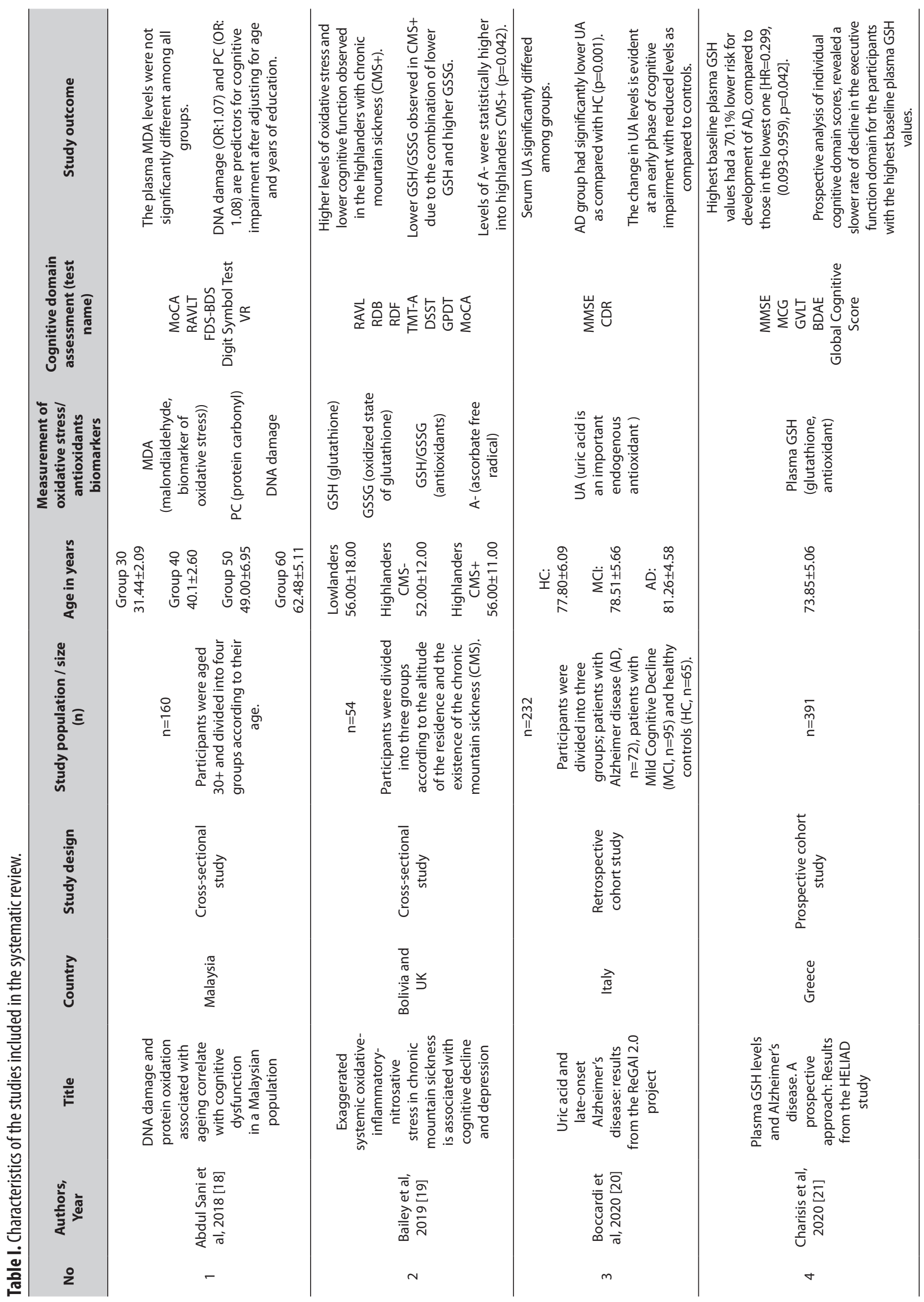




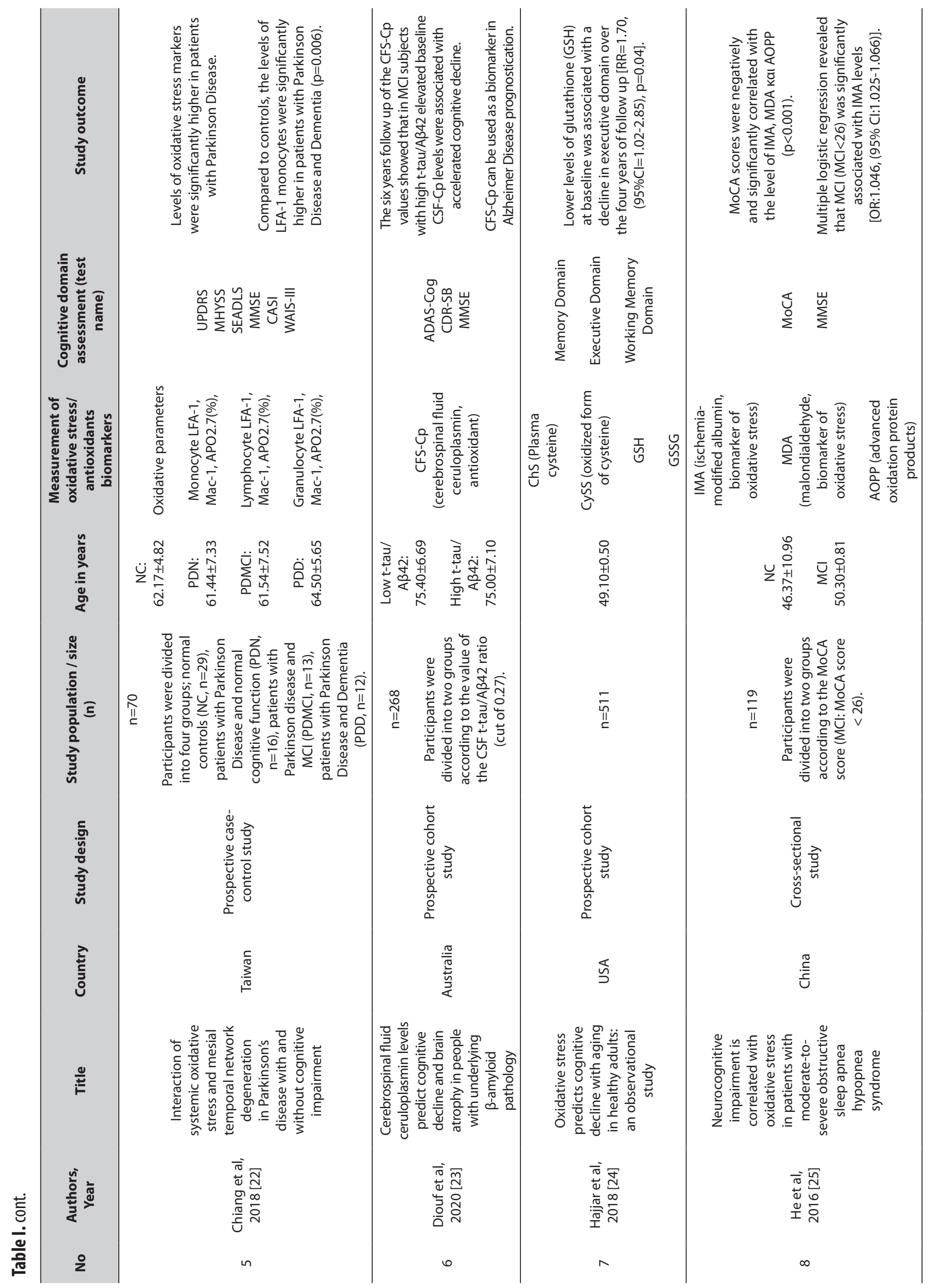




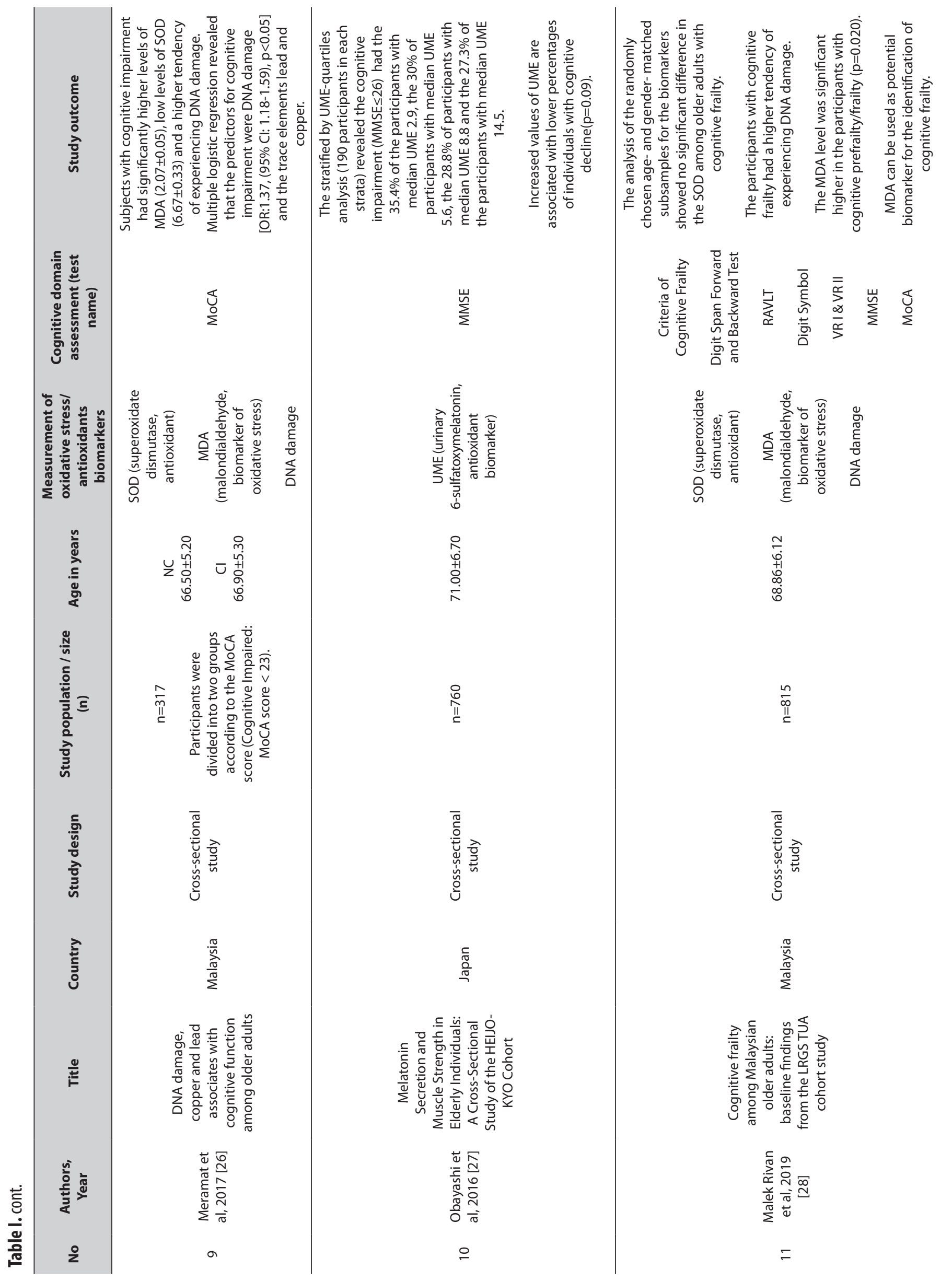




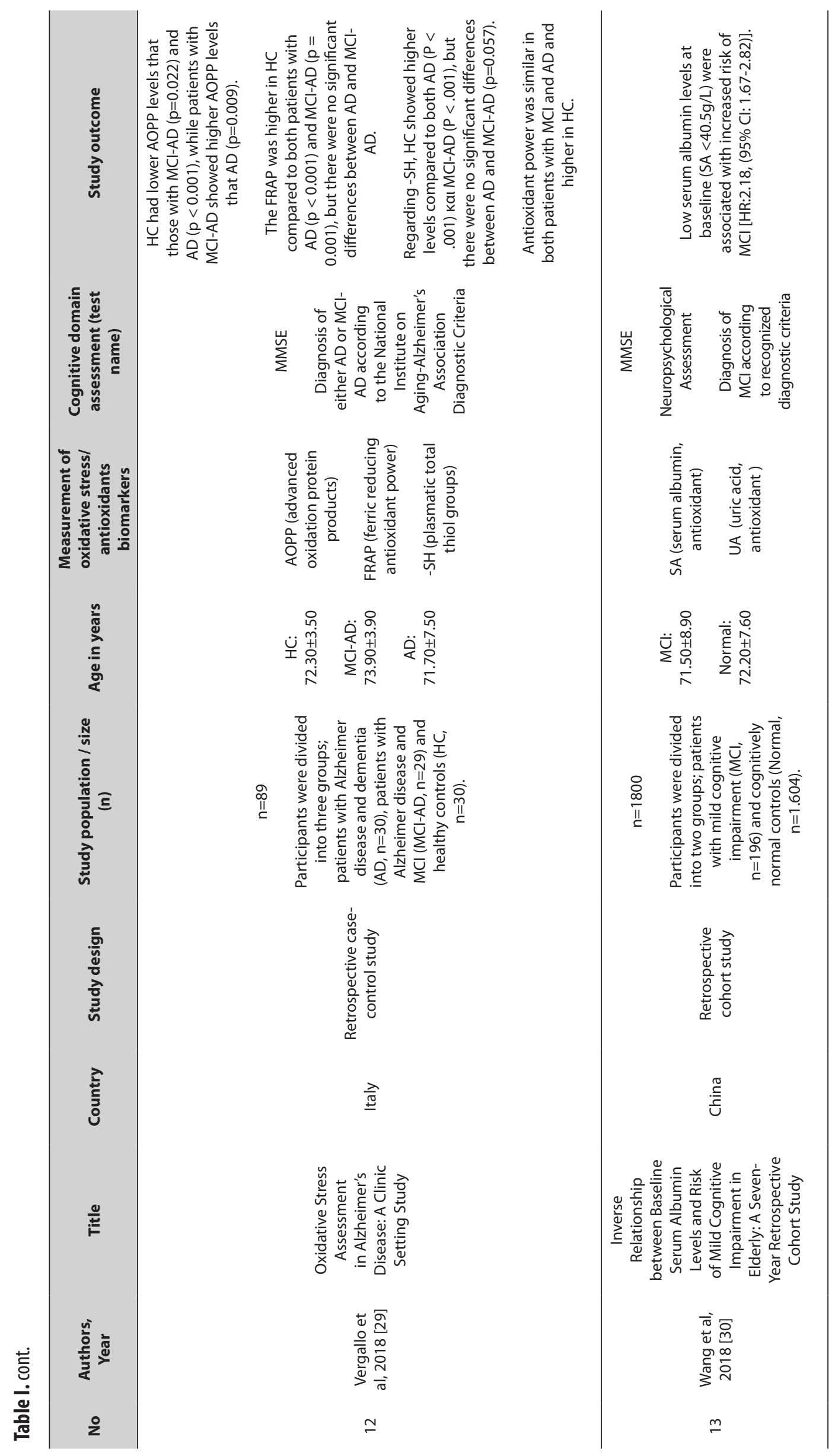


tabases. We chose to include observational studies since we aimed to address the possible association between oxidative stress with cognitive function and highlight its possible contributing role in cognitive impairment. For the review process the keywords were used as follows: ("oxidative stress") AND ("cognitive decline" OR "cognitive dysfunction" OR "cognitive impairment" OR "cognitive deficiency") AND ("observational study" OR "cross sectional study" OR "prospective study" OR "retrospective study" OR "cohort study"). The keywords should be included in the title or summary or keywords of the study. The search was conducted for the years 2016 to 2020 . Due to the inclusion of observational studies the level of evidence of the present systematic review is $2 \mathrm{~A}$.

The eligibility criteria for inclusion studies in the review were: English language-based publication; year of publication 2016 to 2020; population - based sample; observational studies (cohort studies, patient-control studies, cross-sectional studies); measurement of oxidative stress with one or more oxidative biomarkers or antioxidants; assessment of cognitive function with one or more measurement scales.

AXIS was used for the quality assessment of the cross-sectional studies (20 question) [16] and the CASP Checklist was used for the quality assessment of the cohort and case-control studies (12 questions for the cohort studies and 11 questions for the case-control studies) [17].

\section{REVIEW}

From the initial search a total of 93 studies were identified, 23 studies at PubMed and 70 studies at Scopus. Of the 93 studies, 19 were indexed in both databases. Therefore, from the initial search, a total 74 studied were emerged. As it is shown in the flow chart (Fig. 1) of the 74 studies only 13 met the inclusion criteria and were retained for data extraction.

Characteristics of the studies included in the systematic review and details of the studies' populations, methods and results are presented in Table I. The included studies originated from ten different countries: Three studies were Malaysia-based, two were from Italy, two were from China, one study came from Greece, one more from Taiwan, one from Australia, one from USA, one from Japan and finally one study was conducted in two countries, Bolivia and UK.

Regarding the studies' methodology, six are cross-sectional studies [18, 19, 25-28], three are prospective cohort studies $[21,23,24]$, two are retrospective cohort studies $[20,30]$, one is a retrospective case-control study [29], and one is a prospective case-control study [22]. The number of the oxidative stress and anti-oxidant biomarkers measured in the eligible studies ranged from one to nine.

Information on oxidative stress biomarkers was available in eight studies, while information on antioxidant factors was available in ten studies. Regarding oxidative stress biomarkers, information on malondialdehyde (MDA) was available in four studies $[18,25,26,28]$, three studies collected information on DNA damage $[18,26,28]$ and two on oxidized glutathione (GSSG) $[19,24]$ and advanced oxidation protein products (AOPP) $[25,29]$. Other oxidative stress biomarkers, i.e. protein carbonyl (PC), ascorbate free radical (A-), monocyte LFA-1, Mac-1, APO2.7 (\%), lymphocyte LFA-1, Mac-1, APO2.7 (\%), granulocyte LFA1, Mac-1, APO2.7 (\%), oxidized form of cysteine (CySS), ischemia-modified albumin (IMA) and plasmatic total thiol groups $(-\mathrm{SH})$, were measured less frequently.

The antioxidants that measured in the studies that included in the systematic review were glutathione (GSH) in three studies $[19,21,24]$, plasma uric acid (UA) in two studies $[20,30]$, superoxidate dismutase (SOD) in two studies $[26,28]$, and less frequently measured the cerebrospinal fluid ceruloplasmin (CFS-Cp), cysteine (Cys), urinary 6-sulfatoxymelatonin excretion (UME), ferric reducing antioxidant power (FRAP) and serum albumin (SA).

Cognitive function was assessed in all thirteen studies with specific screening tests/scales, while in some of the studies approved diagnostic criteria of cognitive decline or dementia were applied. The most commonly used scales were the Montreal Cognitive Assessment Scale - MoCA, a psychometric test that detects the Mild Cognitive Decline (MCI) and the Mini-Mental State Examination - MMSE, which is a short cognitive assessment test. In total specific areas of cognitive function were assessed, such as memory, executive function, attention, verbal learning ability, speed and ability of cognitive understanding.

Table 1 shows that in the majority of the studies oxidative biomarkers were positively associated with cognitive decline, while antioxidants were associated with better cognitive function.

\section{DISCUSSION}

The aim of this systematic review was to examine the available evidence for the association between oxidative stress and cognitive decline from population-based observational studies that were published during the years 2016-2020. The studies included displayed significant heterogeneity in terms of indicators of oxidative stress and cognitive decline, as well as in methodology, that did not allow us to perform multiple grouping of the results. However, our results indicate that higher levels of oxidative stress may be associated with cognitive decline.

\section{MALONDIALDEHYDE (MDA) AND COGNITIVE DECLINE}

Table 1 shows that the role of MDA in cognitive impairment was examined in four studies $[18,25,26,28]$. MDA is the organic substance that results from lipid peroxidation. It is a biomarker of oxidative stress as it is a measure of the impact of oxygen free radicals on lipids. The cell membrane lipid peroxidation leads to loss of fluidity and elasticity of the membrane, reduced cell function and can even lead to cell rupture and cell death. Lipid peroxidation can contribute to the pathology of many diseases including atherosclerosis, diabetes and Alzheimer's disease.

In only one of the four studies that are included in the systematic review the MDA levels were not associated with the cognitive function. This is a cross-sectional study in 
which the 160 participants were from twenty-five different regions of Malaysia and were divided into four aged groups. Although MDA's value differed statistically between groups, multiple regression showed that MDA is not a predictor of cognitive function [18]. In contrast, in two other studies [18, 25] MDA levels were negatively and significantly correlated with cognitive impairment levels while in one study [26] subjects with cognitive impairment displayed significantly higher levels of MDA. These three studies were cross-sectional and the test that used to assess cognitive function was the MoCA $[25,26,28]$.

\section{GLUTATHIONE AND COGNITIVE DECLINE}

The role of glutathione in cognitive function, either in reduced form (GSH) or in its oxidized form (GSSG), has been investigated in three studies [19,21,24]. Glutathione (GSH) is a tripeptide that is synthesized in cells of the human body and is involved in many biological processes such as in the removal of active oxygen radicals (antioxidant), in the regulation of the redox status of cells, in the regulation of oxidative state of proteins and in regulating the function of the immune system. Reduced GSH is the active form of the tripeptide while its oxidized form (GSSG) is produced after its action.

Glutathione levels decrease with age and its deficiency has been shown to increase vulnerability to damage from oxygen free radicals, thus accelerating oxidation and aging. Low glutathione levels have been linked to many diseases, including cardiovascular, cancer and neurodegenerative diseases, most notably Alzheimer's and Parkinson's disease. The two prospective studies $[21,24]$ that examined the role of glutathione showed that higher glutathione values at baseline are statistically associated with a reduced risk of Alzheimer's disease and better executive function.

\section{PLASMA URIC ACID AND COGNITIVE DECLINE}

Information on the relationship of plasma uric acid to cognitive impairment can be generated from two studies $[20,30]$. Uric acid (UA) is an important endogenous antioxidant and can prevent oxidative stress due to its ability to eliminate reactive oxygen species and act as a pro-oxidant depending on the chemical microenvironment. In human plasma the circulating UA acts as an antioxidant by various mechanisms [20].

In a retrospective study that conducted in Italy 232 elderly people participated. The sample was divided into three groups; elderly with Alzheimer's disease (AD), elderly with mild cognitive impairment (MCI) and healthy elderly. The analysis showed that the elderly with AD had statistically significantly lower UA than healthy seniors. In the whole sample a percentage of $21.12 \%$ had values greater than or equal to $6 \mathrm{mg} / \mathrm{dL}$. After adjusting for confounding factors such as age, sex, BMI and creatinine levels, uric acid levels appeared to be associated with the diagnosis of $\mathrm{AD}$. The data analysis for elderly with mild cognitive impairment showed that changes in UA levels provides evidence of an early cognitive impairment and that this group had lower UA levels in relation to healthy older adults [20].

\section{CONCLUSIONS}

The results of the systematic review showed that the majority of indicators of oxidative stress, and antioxidants may affect the level of cognitive function. Statistically significant associations were observed in many studies between high levels of oxidizing biomarkers and low cognitive ability and function. Antioxidants such as glutathione, uric acid, melatonin, cysteine and peroxide dismutase have been studied in cross-sectional, retrospective and prospective observational studies. The relationship of the presence of antioxidants with better cognitive function was observed in many of them.

The use of specific and approved methods for measuring oxidative stress indicators, as well as the psychometric and neurodiagnostic methods for assessing cognitive function makes the measurable results of studies valid and reliable. However, it is necessary to conduct additional studies either observational or interventional, in order to investigate in depth the mechanisms of action of the variety of biomarkers of oxidative stress in brain function, which should take into account additional factors, such as the duration of oxidation.

\section{REFERENCES}

1. Gray JA. Brain systems that mediate both emotion and cognition. Cognit Emot. 1990;4(3):269-288.

2. Squire LR, Zola-Morgan S. Memory: brain systems and behavior. Trends Neurosc. 1988;11(4):170-175.

3. Lyon GR, Krasnegor NA, McMenamin SMA. Attention, memory, and executive function. J Develop Behav Pediatr. 1996;17(4):278.

4. Bettio LEB, Rajendran L, Gil-Mohapel J. The effects of aging in the hippocampus and cognitive decline. Neurosc Biobehav Rev. 2017;79:66-86.

5. Jongsiriyanyong S, Limpawattana P. Mild cognitive impairment in clinical practice: a review article. Am J Alzh Dis Other Dement. 2018;33(8):500-507.

6. Deary IJ, Corley J, Gow AJ, et al. Age-associated cognitive decline. Brit Med Bull. 2009;92(1):135-152.

7. FisherME,CruickshanksKJ,SchubertCR, etal.Age-related sensoryimpairments and risk of cognitive impairment. J Am Geriatr Soc. 2016;64:1981-1987.

8. Mitchell AJ, Shiri-Feshki M. Rate of progression of mild cognitive impairment to dementia - meta analysis of 41 robust inception cohort studies. Acta Psychiatr Scand. 2009;119(4):252-265.

9. Berr C, Balansard B, Arnaud J, Roussel A, et al. Cognitive decline is associated with systematic oxidative stress: The EVA study. J Am Geriatr Soc. 2015;48(10):1285-1291.

10. Sies H, BerndtC, Jones D. Oxidativestress. An Rev Biochem. 2017;86:715-748.

11. Mancuso M, Orsucci D, LoGerfo A, et al. Oxidative stress biomarkers in mitochondrial myopathies basally and after cysteine donor supplementation. J Neurol. 2010;257:774-781.

12. Berlett B, Stadtman ER. Protein oxidation in aging, disease, and oxidative stress. The journal of biochemical chemistry. 1997;272(33):20313-20316.

13. Mao P. Oxidative stress and its clinical application in dementia. J Neurodegenerat Dis. 2013.

14. Cervellati C, Romani $A$, et al. Oxidative balance, homocysteine, and uric acid levels in older patients with late onset Alzheimer's disease or vascular dementia. J Neurol Sci. 2014;337(1-2):156-161. 
15. Bergamm CM, Gambetti S, Dondi A, Cervellati C. Oxygen, reactive oxygen species and tissue damage. Cur Pharmaceut Des. 2004;10(14):1611-1626.

16. Downess MJ, Brennan ML, Williams HC, Dean RS. Development of a critical appraisal tool to assess the quality of cross-sectional studies (AXIS). BMJ Open. 2016;6e011458.

17. CASP Critical Appraisal Skills Programme [Internet]. Oxford: CASP UK. [cited 2020 Jan 28]. https://casp-uk.net/

18. Abdul Sani NF, Damanhuri AHA, Hamzah Al, et al. DNA damage and protein oxidation associated with ageing correlate with cognitive dysfunction in a Malaysian population. Fee Rad Res. 2018;52(9):1000-1009.

19. Bailey DM, Brugniaaux JV, Filipponi T, et al. Exaggerated systemic oxidativeinflammatory-nitrosative stress in chronic mountain sickness is associated with cognitive decline and depression. J Physiol. 2019;592(2):611-629. doi: 10.1113/JP276898

20. Boccardi V, Carlino S, Marinelli E, et al. Uric acid and lateonset Alzheimer's disease: results from the ReGAI 2.0 project. Aging Clin Experiment Res. 2020.

21. Charisis S, Ntanasi E, Yannakoulia M, etal. Plasma GSH levels and Alzheimer's disease. A prospective approach: Results from the HELIAD study. Free Rad Biol Med. 2020.

22. Chiang PL, Chen HL, Lu CH, et al. Interaction of systemic oxidative stress and mesial temporal network degeneration in Parkinson's disease with and without cognitive impairment. J Neuroinflam. 2018;15:281.

23. Diouf I, Bush Al, Ayton S. Cerebrospinal fluid ceruloplasmin levels predict cognitive decline and brain atrophy in people with underlying $\beta$-amyloid pathology. Neurobiol Dis. 2020.

24. Hajjar I, Hayek SS, Goldstein FC, Martin G, Jones GM, Ouyyumi A. Oxidative stress predicts cognitive decline with aging in healthy adults: an observational study. J Neuroinflam. 2018;15:17.

25. He Y, Chen R, Wang J, Pan W, Sun Y, Han F, Wang Q, Liu C. Neurocognitive impairment is correlated with oxidative stress in patients with moderateto-severe obstructive sleep apnea hypopnea syndrome. Respirat Med. 2016;120:25-30.

26. Meramat A, Rajab NF, Shahar S, Sharif RA. DNA damage, copper and lead associates with cognitive function among older adults. J Nutr Health Aging. 2017;21(5):539-545. doi: 10.1007/s12603-016-0759-1

27. Obayashi K, Saeki K, Maegawa T, et al. Melatonin Secretion and Muscle Strength in Elderly Individuals: A Cross-Sectional Study of the HEIJO-KYO Cohort. J Gerontol. Series A, Biol Sci Med Sci. 2016;71(9):1235-1240. doi: 10.1093/gerona/glw030
28. Malek Rivan NF, ShaharS, Rajab NF, et al. Cognitive frailty among Malaysian older adults: baseline findings from the LRGS TUA cohort study. Clin Interv Aging. 2019;14:1343-4352. doi: 10.2147/CIA.S211027

29. Vergallo A, Giampietri L, Baldacci F, et al. Oxidative Stress Assessment in Alzheimer's Disease: A Clinic Setting Study. Am J Alzh Dis Dement. 2018;33(1):35-41. doi: 10.1177/1533317517728352

30. Wang L,WangF,LiuJ,Zhang Q, LeiP.Inverse Relationship between BaselineSerum Albumin Levels and Risk of Mild Cognitive Impairment in Elderly: A Seven-Year RetrospectiveCohortStudy.TJEM.2018;246(1):51-57.doi:10.1620/tjem.246.51

\section{ORCID and contributionship:}

Ioanna V. Papathanasiou: 0000-0002-8874-8085 A,B,D

Evangelos C. Fradelos: 0000-0003-0244-9760 ${ }^{B}$

Foteini Malli: 0000-0002-7595-6715 ${ }^{B}$

Ioannis Stefanidis: 0000-0002-5515-8089 ${ }^{\mathrm{E}}$

Elias Zintzaras: 0000-0002-4450-0125 ${ }^{\mathrm{E}}$

Chrysoula Doxani: 0000-0003-1062-1364 A,E-F

\section{Conflict of interest:}

The Authors declare no conflict of interest

\section{CORRESPONDING AUTHOR loanna V. Papathanasiou}

Faculty of Nursing, University of Thessally, Gaiopolis Campus, Larissa - Trikala Ring Road, 41500, Larissa, Greece tel: 00306977637933

e-mail: iopapathanasiou@yahoo.gr

Received: 02.05 .2021

Accepted: 30.07 .2021

A - Work concept and design, B - Data collection and analysis, C - Responsibility for statistical analysis, D-Writing the article, $\mathbf{E}$-Critical review, $\mathbf{F}$ - Final approval of the article 